\title{
High-Resolution Magnifying Video Endoscopy in Primary Intestinal Lymphangiectasia: a New Role for Endoscopy?
}
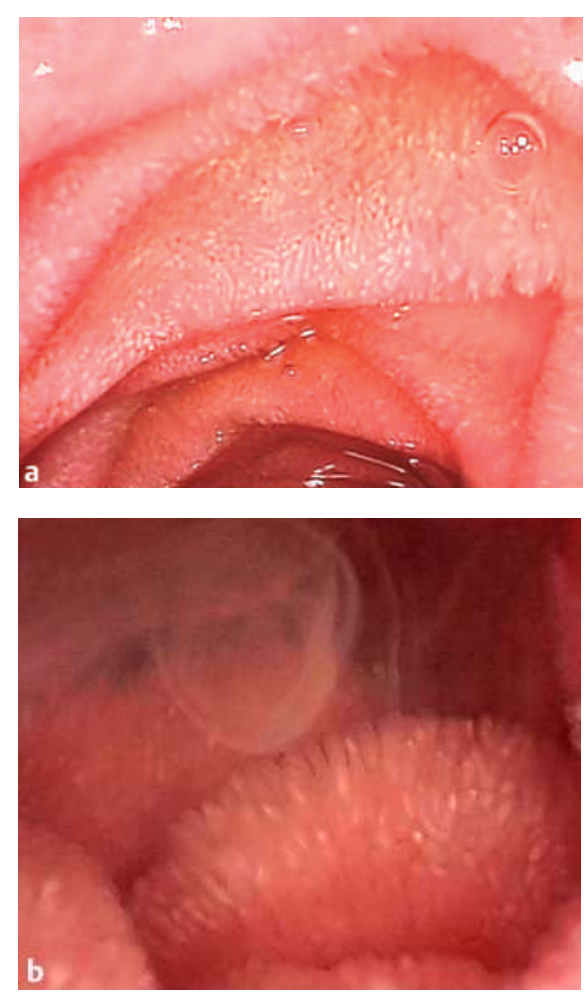

Figure 1 A 21-year-old woman, with a 14month history of recurrent diarrhea, steatorrhea, hypoproteinemia and peripheral oedema, underwent upper gastrointestinal endoscopy. A Fuji EG-485ZH video endoscope was used, which is capable of providing high-resolution images (because of its 850000 -pixel chip) and up to $2 \times$ magnification. In the second part of the duodenum the mucosal surface had an appearance resembling rice grains (a), and diffuse fluid, which was producing a smoke-like mist, was observed (b).

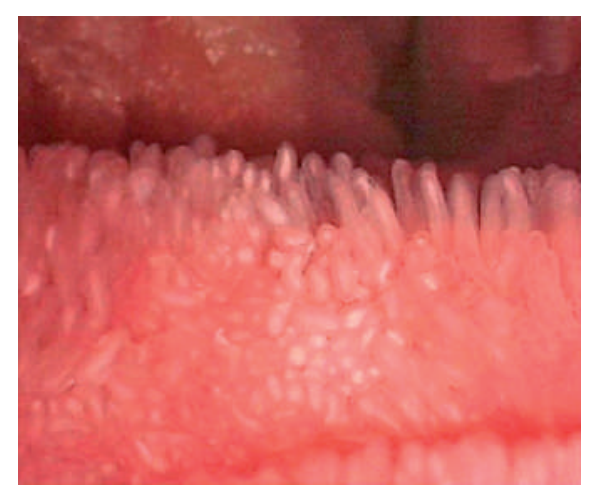

Figure 2 Under magnification, the finger-like processes were seen to resemble small white lights. A final diagnosis of primary intestinal lymphangiectasia was made. With the newgeneration video endoscope used in this case we were able to obtain images never seen before, images showing dilated capillary vessels in intestinal villi and the loss of lymph fluid into the gastrointestinal tract.
G. Cammarota, R. Cianci, G. Gasbarrini Department of Internal Medicine and Gastroenterology, Catholic University of Rome, Italy

\section{Corresponding Author}

\section{G. Cammarota, M.D.}

Istituto di Medicina Interna,

Policlinico Universitario “A. Gemelli”

Largo A. Gemelli, 8

00168 Roma

Italia

Fax: $\quad+39-06-35502775$

E-mail: gcammarota@rm.unicatt.it 